\title{
EXCEPTIONAL BLOWUP SOLUTIONS TO QUASILINEAR WAVE EQUATIONS II*
}

\author{
SERGE ALINHAC ${ }^{\dagger}$
} solutions

Key words. Quasilinear wave equations, blowup, linearly degenerate eigenvalue, unstable

AMS subject classifications. $35 \mathrm{~L} 40$

1. Introduction. This Note is a continuation of the paper "Exceptional Blowup Solutions to Quasilinear Wave Equations" [1]. In this previous paper, we constructed, for some quasilinear wave equations, solutions blowing up at the origin like $t^{-2}$, which we considered to be an exceptional rate (the standard one in this context being $t^{-1}$, see $[6]$ ). We were encouraged by questions of the Referee (whom we thank) to investigate more precisely the stability of such solutions (an issue vaguely touched upon in [1]). It turns out that, depending on the perturbation of the Cauchy data, we can make the singularity of the solution either disappear, or go back to the generic $t^{-1}$ case.

Since this paper is dedicated to M. S. Baouendi, we are happy to underline the similarity in spirit between previous constructions of conterexamples [7], [8], and the present work : in both cases, the insight is obtained through a careful self-contained construction.

2. Notation and main result. The notation and the framework is the same as in [1]. For simplicity, we restrict our attention to $n=2$, and do not handle the 1D case (though it is straightforward). Thus the variables and dual variables are

$$
x=\left(x_{1}, x_{2}, x_{3}\right), y=x_{2}, t=x_{3}, \xi=\left(\xi_{1}, \xi_{2}, \xi_{3}\right), \eta=\xi_{2}, \tau=\xi_{3} .
$$

We consider a quasilinear wave equation with real analytic coefficients

$$
P(u)=\Sigma p_{i j}(\partial u) \partial_{i j}^{2} u=0, p_{i j}=p_{j i}, p_{3,3}=1
$$

We denote here

$$
\partial u=\left(\partial_{1} u, \partial_{2} u, \partial_{3} u\right), p(\partial u ; \xi)=\Sigma p_{i j}(\partial u) \xi_{i} \xi_{j}
$$

We assume given a point $(\overline{\partial u}, \bar{\xi})$ where

$$
p(\overline{\partial u} ; \bar{\xi})=0,\left(\partial_{\tau} p\right)(\overline{\partial u} ; \bar{\xi}) \neq 0, \bar{\xi}_{1}=-1
$$

and the frozen operator $\Sigma p_{i j}(\overline{\partial u}) \partial_{i j}^{2}$ is strictly hyperbolic with respect to $t$. Noting $D_{j} p=\partial_{\left(\partial_{j} u\right)} p$, we assume moreover that the given point is linearly degenerate, that is

$$
(\bar{\xi} . D) p(\overline{\partial u} ; \bar{\xi})=0
$$

\footnotetext{
*Received June 20, 2006; accepted for publication September 13, 2006.

†Département de Mathématiques, Université Paris-Sud, 91405 Orsay, France (serge.alinhac@ math.u-psud.fr).
} 
In this Note, our aim is to improve the results of [1] by constructing a family of solutions $u^{\epsilon}$, containing the solution constructed in [1] as $u^{0}$, and such that,

i) For $\epsilon>0$, the solution does not blow up,

ii) For $\epsilon<0$, the solution blows up at time $t_{\epsilon}$ like $\left(t_{\epsilon}-t\right)^{-1}$.

The assumptions of our main result below are the same as those of Theorem 2.3 of [1].

Theorem. Assume that, at the given point $(\overline{\partial u}, \bar{\xi}), \partial_{\eta} p \neq 0$, and one of the following three quantities is not zero:

$$
\begin{gathered}
-\left(D_{2} p\right)\left((\bar{\xi} D) \partial_{\eta} p+D_{2} p\right)-1 / 2\left(\partial_{\eta}^{2} p\right)(\bar{\xi} D)^{2} p+\left(\partial_{\eta} p\right)(\bar{\xi} D) D_{2} p, \\
-\left(D_{3} p\right)\left((\bar{\xi} D) \partial_{\eta} p+D_{2} p\right)-\left(D_{2} p\right)\left((\bar{\xi} D) \partial_{\tau} p+D_{3} p\right)- \\
\quad-\left(\partial_{\tau}^{2} p\right)(\bar{\xi} D)^{2} p+\left(\partial_{\tau} p\right)(\bar{\xi} D) D_{2} p+\left(\partial_{\eta} p\right)(\bar{\xi} D) D_{3} p \\
-\left(D_{3} p\right)\left((\bar{\xi} D) \partial_{\tau} p+D_{3} p\right)-(\bar{\xi} D)^{2} p+\left(\partial_{\tau} p\right)(\bar{\xi} D) D_{3} p .
\end{gathered}
$$

Then there exist

a. A domain $D$ defined by

$$
-t_{1} \leq t \leq t_{2}, x_{1}^{2}+x_{2}^{2} \leq(R-k t)^{2}, t_{1}>0, t_{2}>0, R>0, k>0 .
$$

We call the disk $t=-t_{1},\left\|\left(x_{1}, x_{2}\right)\right\| \leq R+k t_{1}$ the base of $D$.

b. A family of solutions $u^{\epsilon}$ (depending continuously on $\epsilon$ along with its derivatives), defined for $\epsilon$ close to zero such that

i) For $\epsilon=0$, let

$$
D_{0}=D \cap\{t<0\} .
$$

Then $u^{0} \in C^{1}\left(\bar{D}_{0}\right), u^{0}$ is analytic in $\bar{D}_{0}$ away from the origin, $D_{0}$ is an influence domain of its base, and $\partial^{2} u^{0}$ blows up at the origin like $(-t)^{-2}$, as explained precisely in [1].

ii) For $\epsilon>0, u^{\epsilon}$ is defined and analytic in $D$,

iii) For $\epsilon<0$ and some $t_{\epsilon}<0$, let

$$
D_{\epsilon}=D \cap\left\{t<t_{\epsilon}\right\} .
$$

Then $u^{\epsilon} \in C^{1}\left(\bar{D}_{\epsilon}\right), u^{\epsilon}$ is analytic in $\bar{D}_{\epsilon}$ away from the origin, $D_{\epsilon}$ is an influence domain of its base, and $\partial^{2} u^{\epsilon}$ blows up at a point $m_{\epsilon}=\left(p_{\epsilon}, t_{\epsilon}\right)$, close to zero, like $\left(t_{\epsilon}-\right.$ $t)^{-1}$ (more precisely, $m_{\epsilon}$ is a geometric blowup point of cusp type, in the terminology of [2], [3]).

REMARK 1. We cannot describe which modifications of the Cauchy data on $t=-t_{1}$ lead to which singularities for the solution. What we do is show that some modifications of the data make the singularity disappear, while some other transform the singularity back to the generic type.

REMARK 2. What we call "geometric blowup of cusp type", or more rapidly "generic blowup", is indeed stable, as shown in [5] in the more general context of quasilinear symmetric systems.

This Note is closely related to [1] : though the statements and the strategy of the proof are understandable without [1], the actual details are based on computations of [1], and can only be understood in connection with [1]. 


\section{Proof of the main result.}

3.1. Outline of the strategy. We recall here the basic facts from [1], section 3 , keeping the same notation. For a broader introduction to the concepts and tools used here, see for instance [2], [3], [4]. We introduce a change of variables $\Phi$ (depending on a still unknown function $\phi$ )

$$
(s, y, t) \mapsto\left(x_{1}=\phi(s, y, t), y, t\right),
$$

and set

$$
w(s, y, t)=u(\phi(s, y, t), y, t), v(s, y, t)=\left(\partial_{1} u\right)(\phi(s, y, t), y, t)
$$

Setting

$$
\bar{\partial}=\left(0, \partial_{y}, \partial_{t}\right), \hat{\phi}=\left(-1, \partial_{y} \phi, \partial_{t} \phi\right)
$$

we define now

$$
\begin{gathered}
\mathcal{A}=w_{s}-v \phi_{s}, \\
\mathcal{E}=\Sigma p_{i j}\left(v, w_{y}-v \phi_{y}, w_{t}-v \phi_{t}\right) \hat{\phi}_{i} \hat{\phi}_{j}, \\
\mathcal{R}=\Sigma p_{i j}\left(v, w_{y}-v \phi_{y}, w_{t}-v \phi_{t}\right)\left[\bar{\partial}_{i j}^{2} w-v \bar{\partial}_{i j}^{2} \phi-\left(\hat{\phi}_{i} \bar{\partial}_{j} v+\hat{\phi}_{j} \bar{\partial}_{i} v\right)\right] .
\end{gathered}
$$

Using the formula for the first and second order derivatives of $u$ in terms of $(v, \phi, w)$, we see easily that

$$
(P u)(\Phi)=\frac{\mathcal{E}}{\phi_{s}}+\mathcal{R} .
$$

Hence we associate to $P$ the blowup system on $(\phi, v, w)$

$$
\mathcal{A}=0, \mathcal{E}=0, \mathcal{R}=0 \text {. }
$$

Rotating the variables by

$$
T=s+t, S=t-s, y=y,
$$

the write the subsystem $\mathcal{A}=0, \mathcal{E}=0$ as

$$
w_{T}=w_{S}-2 v \phi_{S}+v \lambda, \phi_{T}=-\phi_{S}+\lambda
$$

where

$$
\lambda \equiv \lambda\left(v, w_{y}-v \phi_{y}, 2\left(w_{S}-v \phi_{S}\right) ;-1, \phi_{y}\right) .
$$

Using these equations, and introducing the new unknowns $\phi_{S}, \phi_{y}, w_{S}, w_{y}$, we can view the blowup system as a fully nonlinear first order system in the unknowns $v, \phi_{S}, \phi_{y}, w_{S}, w_{y}$, resolved with respect to the $T$-derivative. Our aim is to construct, using the Cauchy-Kovalevski theorem, a family of (smooth) solutions (near the origin) of the blowup system.

Heuristically, the construction of the family $u^{\epsilon}$ is based on a simple geometric deformation argument which goes as follow : we will construct $\phi$ such that, very roughly,

$$
\phi_{s}=\epsilon+s^{2}+y^{2}+t^{2} .
$$

Then

i) For $\epsilon=0$, we have the exceptional blowup described in [1], 
ii) For $\epsilon>0$, we have no blowup at all in a fixed neighbourhood of the origin,

iii) For $\epsilon<0, \phi_{s}=0$ at the point $M_{\epsilon}=\left(0,0, t_{\epsilon}=-(-\epsilon)^{1 / 2}\right)$ and is stricly positive for $t<t_{\epsilon}$.

3.2. Choice of the jets at the origin. We modify now the construction of [1] by choosing a larger family of jet conditions at the origin. We still impose

$$
\phi_{y}=\bar{\eta},\left(v, w_{y}-v \phi_{y}, 2\left(w_{S}-v \phi_{S}\right)\right)=\bar{\partial} u
$$

but we take now, for $\epsilon$ small enough (positive and negative)

$$
\lambda-2 \phi_{S}=\epsilon
$$

Remark that, from Lemma 3.2 de [1], $T=0$ is still non characteristic for the blowup system. Note also that, at the origin, $\Lambda$ is no longer zero, but only $O(\epsilon)$.

We choose now the values of the blocks $B_{y y}, B_{S y}, B_{S S}$ just as before for $\epsilon=0$, in order to ensure that $Q \neq 0$. With $\phi_{y y}(0)=0$, we then choose successively $\phi_{S y}(0)$ and $\phi_{S S}(0)$ to obtain from Lemma 4.1

$$
F_{y}(0)=0, F_{S}(0)=0
$$

but now of course $F_{T}$ need not be zero, but only $O(\epsilon)$.

For the third order jets, we proceed exactly as in 4.4 .3 of [1], choosing the blocks $B_{S S S}, B_{S S y}$, etc. to be zero ; then

$$
\phi_{y y y}(0)=0, A=-\phi_{S y y}(0)>>0,2 \phi_{S S y}(0)=-\partial_{\eta} \lambda A, \phi_{S S S}=-B=-\frac{1}{2}\left(\partial_{\eta} \lambda\right)^{2} A .
$$

Since, according to Lemma 4.2 of [1], second order derivatives of $F$ involve also second order derivatives of $v$, we fix for clarity, say,

$$
v_{y y}(0)=v_{S y}(0)=v_{S S}(0)=0 .
$$

We fix now the value of $A$ as for the case $\epsilon=0$. For small enough $\epsilon$, the hessian of $F$ at the origin will still be positive definite.

3.3. Uniformity. Once the jets of $\phi, v, w$ are fixed as above, we solve the blowup system taking for $\phi, v, w$ polynomials of degree respectively $3,2,3$ with these jets. Using a precise version of the Cauchy-Kovalevski theorem (for instance that of Baouendi and Goulaouic [9]), we see that we obtain a family of solutions defined in a fixed neighbourhood of the origin, whose derivatives are continuous in $\epsilon$.

Two facts remain to be proved :

i) If $\epsilon>0$, there is a fixed neighbourhood of 0 where $F$ remains positive.

ii) If $\epsilon<0$, there is a point close to 0 where $F=0$, and the corresponding solution blows up.

4. Case $\epsilon>0$. This is the easy case. In fact, expanding $F$ by Taylor up to second order and using the fact that the hessian is uniformly positive definite in a fixed neighbourhood of the origin, we obtain, for some $C^{\prime}>0$,

$$
F(S, y, T) \geq F(0)-C \epsilon\|(S, y, T)\|+C^{\prime}\left(S^{2}+y^{2}+T^{2}\right), F(0)=\epsilon .
$$


Hence $F>0$ for $\epsilon<4 C^{\prime} / C^{2}$.

5. Case $\epsilon<0$.

a. For given $(\epsilon, T)$, we look first for a point where $\phi_{s s}=\phi_{s y}=0$, that is

$$
F_{T}-F_{S}=0, F_{y}=0
$$

For $\epsilon=0$ and $T=0$, the origin is such a point. To apply the implicit function theorem, it is enough to check that, at the origin,

$$
d=F_{y y}\left(F_{S T}-F_{S S}\right)-F_{S y}\left(F_{T y}-F_{S y}\right) \neq 0 .
$$

With the above choices of jets, we have

$$
d=-\delta+F_{S T} F_{y y}-F_{S y} F_{T y}=-2\left(\partial_{\eta} \lambda\right)^{2} A^{2}+O(A)
$$

We can always assume that $A$ has been already chosen so big that $d \neq 0$. So we have now functions $S(\epsilon, T)$ and $y(\epsilon, T)$, smoothly defined near zero, such that $\phi_{s s}=\phi_{s y}=0$ at the point

$$
M(\epsilon, T)=(S=S(\epsilon, T), y=y(\epsilon, T), T)
$$

b. We set now

$$
\tilde{F}(\epsilon, T)=F(S(\epsilon, T), y(\epsilon, T), T),
$$

and look for $T=T_{\epsilon}$ such that

$$
\tilde{F}\left(\epsilon, T_{\epsilon}\right)=0
$$

LEMMA 5.1.For $\epsilon=0, T=0$, if we choose $\left(v_{T}-v_{S}\right) Q>0$ and $v_{T}-v_{S}$ small enough, then

$$
\tilde{F}_{T T}(0,0)>0
$$

Proof. We have

$$
\tilde{F}_{T}(\epsilon, T)=F_{T}+F_{S} S_{T}+F_{y} y_{T} .
$$

Since at $M(\epsilon, T), F_{T}=F_{S}$ and $F_{y}=0$, we obtain

$$
F_{T}=F_{S}=(1 / 2)\left(v_{T}-v_{S}\right) \Lambda+* F
$$

hence

$$
\begin{gathered}
\tilde{F}_{T}=\left(S_{T}+1\right) F_{S}=(1 / 2)\left(S_{T}+1\right)\left(v_{T}-v_{S}\right) \Lambda+* F \\
\tilde{F}_{T T}(0)=(1 / 2)\left(S_{T}+1\right)\left(v_{T}-v_{S}\right)\left(\partial_{T} \Lambda+\partial_{S} \Lambda S_{T}+\partial_{y} \Lambda y_{T}\right) .
\end{gathered}
$$

Now, differentiating the identities which define $S$ and $y$, we obtain

$$
\begin{gathered}
F_{T T}-F_{S T}+S_{T}\left(F_{T S}-F_{S S}\right)+y_{T}\left(F_{T y}-F_{S y}\right)=0, \\
F_{y T}+S_{T} F_{y S}+y_{T} F_{y y}=0 .
\end{gathered}
$$

Replacing $F_{T T}, F_{T S}, F_{T y}$ using the formula of Lemma 4.2 of [1], we see that the equalities are satisfied, up to some term $O\left(v_{T}-v_{S}\right)$, if we take $S_{T}=1, y_{T}=-\partial_{\eta} \lambda$. Hence

$$
S_{T}=1+O\left(v_{T}-v_{S}\right), y_{T}=-\partial_{\eta} \lambda+O\left(v_{T}-v_{S}\right)
$$




$$
\Lambda_{T}+S_{T} \Lambda_{S}+y_{T} \Lambda_{y}=Q+O\left(v_{T}-v_{S}\right) .
$$

This proves the claim.

LEMMA 5.2. There exists, for $\epsilon<0$ small enough, a smooth function $T_{\epsilon}=$ $T\left((-\epsilon)^{1 / 2}\right)$, such that

$$
\begin{gathered}
T_{\epsilon}=-c(-\epsilon)^{1 / 2}+O(\epsilon), c>0, \\
\tilde{F}\left(\epsilon, T_{\epsilon}\right)=0 .
\end{gathered}
$$

Proof. Let us write the Taylor expansion of $\tilde{F}$ at $(0,0)$ :

$\tilde{F}(\epsilon, T)=\tilde{F}(0,0)+\epsilon \partial_{\epsilon} \tilde{F}(0,0)+T \tilde{F}_{T}(0,0)+O\left(\epsilon^{2}\right)+O(\epsilon T)+\left(T^{2} / 2\right) \tilde{F}_{T T}(0,0)+O\left(T^{3}\right)$.

Since

$$
\tilde{F}(0,0)=0, \tilde{F}_{T}(0,0)=0, \partial_{\epsilon} \tilde{F}(0,0)=\left(\partial_{\epsilon} F\right)(0,0)+* F_{S}(0,0)+* F_{y}(0,0)=1,
$$

we get

$$
\tilde{F}(\epsilon, T)=\epsilon(1+O(\epsilon)+O(T))+\left(T^{2} / 2\right) \tilde{F}_{T T}(0)(1+O(T)) .
$$

Introducing $\mu=(-\epsilon)^{1 / 2}$ and applying Morse's Lemma, we obtain the claim, thanks to Lemma 5.1.

We now finish the proof of the Theorem. Let $M_{\epsilon}$ be the point

$$
M_{\epsilon}=M\left(\epsilon, T_{\epsilon}\right)=\left(S\left(\epsilon, T_{\epsilon}\right), y\left(\epsilon, T_{\epsilon}\right), T_{\epsilon}\right)
$$

The $t=t_{\epsilon}$ coordinate of this point is

$$
2 t=S\left(\epsilon, T_{\epsilon}\right)+T_{\epsilon}=2 T_{\epsilon}+O(\epsilon),
$$

and is negative for $\epsilon$ small enough. Consider now the set $\phi_{s}=F=0$; in a small enough neighbourhood of the origin, it is a compact submanifold. At a point of this submanifold where $t$ is minimum, we necessarily have

$$
\phi_{s s}=\phi_{s y}=0
$$

But, from the above considerations, we know that there is only one such point, namely $M_{\epsilon}$. Since, for $t=t_{\epsilon}, \phi_{s}$ has at $M_{\epsilon}$ a critical point with definite positive hessian, it is non negative then, and $\phi_{s}>0$ for $t<t_{\epsilon}$. Hence the image $m_{\epsilon}$ by $\Phi$ of the point $M_{\epsilon}$ corresponds to a blowup point of cusp type, as usual, for the solution of the equation.

\section{REFERENCES}

[1] Alinhac S., Exceptional Blowup Solutions to Quasilinear Wave Equations, to appear, Int. Math. Res. Notices, (2006).

[2] Alinhac S., Blowup for Nonlinear Hyperbolic Equations, Progr. Nonlinear Differential Equations Appl. 17, Birkhäuser Boston, Boston MA, (1995).

[3] Alinhac S., A minicourse on global existence and blowup of classical solutions to multidimensional quasilinear wave equations, Journées "Equations aux Dérivées partielles" (Forges les Eaux, 2002), Université de Nantes, Nantes, 2002. www.math.sciences.univnantes.fr/edpa. 
[4] Alinhac S., Explosion géométrique pour des systèmes quasi-linéaires, Amer. J. Math., 117 (1995), pp. 987-1017.

[5] AlinhaC S., Stability of geometric blowup, Arch. Rat. Mech. Analysis, 150 (1999), pp. 97125.

[6] Alinhac S., Remarks on the blowup rate of classical solutions to quasilinear multidimensional hyperbolic systems, J. Math. Pure Appl., 79 (2000), pp. 839-854.

[7] Alinhac S. and Baouendi M. S., A counterexample to strong uniqueness for Schrödinger's type partial differential equations, Comm. Part. Diff. Eq., 19 (1994), pp. 1727-1733.

[8] Alinhac S. And Baouendi M. S., A non uniqueness result for operators of principal type, Math. Zeitsch., 220 (1995), pp. 561-568.

[9] Baouendi M. S. And Goulaouic C., Remarks on the abstract form of nonlinear CauchyKovalevsky theorems, Comm. Part. Diff. Eq., 2 (1977), pp. 1151-1162. 
